\title{
Comparative analysis of RNA regulatory elements of amino acid metabolism genes in Actinobacteria
}

\author{
Alexander V Seliverstov ${ }^{1}$, Harald Putzer ${ }^{2}$, Mikhail S Gelfand ${ }^{* 1}$ and \\ Vassily A Lyubetsky ${ }^{1}$
}

Address: ${ }^{1}$ Institute for Information Transmission Problems, RAS, Bolshoi Karetny pereulok 19, Moscow, 127994, Russia and ${ }^{2}$ Institut de Biologie Physico-Chimique, CNRS UPR9073, 13, rue P. et M. Curie, 75005 Paris, France

Email: Alexander V Seliverstov - slvstv@iitp.ru; Harald Putzer - Harald.Putzer@ibpc.fr; Mikhail S Gelfand* - gelfand@iitp.ru;

Vassily A Lyubetsky - lyubetsk@iitp.ru

* Corresponding author

Published: 03 October 2005

BMC Microbiology 2005, 5:54 doi:10.1 186/147|-2180-5-54
Received: 01 April 2005

Accepted: 03 October 2005

This article is available from: http://www.biomedcentral.com/I47/-2/80/5/54

(C) 2005 Seliverstov et al; licensee BioMed Central Ltd.

This is an Open Access article distributed under the terms of the Creative Commons Attribution License (http://creativecommons.org/licenses/by/2.0), which permits unrestricted use, distribution, and reproduction in any medium, provided the original work is properly cited.

\begin{abstract}
Background: Formation of alternative structures in mRNA in response to external stimuli, either direct or mediated by proteins or other RNAs, is a major mechanism of regulation of gene expression in bacteria. This mechanism has been studied in detail using experimental and computational approaches in proteobacteria and Firmicutes, but not in other groups of bacteria.

Results: Comparative analysis of amino acid biosynthesis operons in Actinobacteria resulted in identification of conserved regions upstream of several operons. Classical attenuators were predicted upstream of trp operons in Corynebacterium spp. and Streptomyces spp., and trpS and leuS genes in some Streptomyces spp. Candidate leader peptides with terminators were observed upstream of ilvB genes in Corynebacterium spp., Mycobacterium spp. and Streptomyces spp. Candidate leader peptides without obvious terminators were found upstream of cys operons in Mycobacterium spp. and several other species. A conserved pseudoknot (named LEU element) was identified upstream of leuA operons in most Actinobacteria. Finally, T-boxes likely involved in the regulation of translation initiation were observed upstream of ileS genes from several Actinobacteria.
\end{abstract}

Conclusion: The metabolism of tryptophan, cysteine and leucine in Actinobacteria seems to be regulated on the RNA level. In some cases the mechanism is classical attenuation, but in many cases some components of attenuators are missing. The most interesting case seems to be the leuA operon preceded by the LEU element that may fold into a conserved pseudoknot or an alternative structure. A LEU element has been observed in a transposase gene from Bifidobacterium longum, but it is not conserved in genes encoding closely related transposases despite a very high level of protein similarity. One possibility is that the regulatory region of the leuA has been co-opted from some element involved in transposition. Analysis of phylogenetic patterns allowed for identification of MLI 624 of M. leprae and its orthologs as the candidate regulatory proteins that may bind to the LEU element. T-boxes upstream of the ileS genes are unusual, as their regulatory mechanism seems to be inhibition of translation initiation via a hairpin sequestering the Shine-Dalgarno box. 


\section{Background}

Formation of alternative structures in 5'-leader regions of mRNAs is emerging as a major mechanism of gene regulation. There exist several possible variants of this mechanism whose common feature is the competition between two structures, one of which represses gene expression via premature termination of transcription or inhibition of translation initiation (reviewed in [1-6]). The energetically or kinetically more favourable structure forms by default, whereas the other one is stabilized by binding of a regulatory protein, tRNA, or a small cofactor, or is formed co-transcriptionally, as in classical attenuators.

RNA regulatory elements have been studied mainly in gamma-proteobacteria (Escherichia coli) and firmicutes (Bacillus subtilis). Computational analysis also has been mainly restricted to proteobacteria $[7,8]$ and firmicutes [912]. Recently a new class of regulatory elements, riboswitches, has been described. These elements are highly conserved and were found in all major taxa of bacteria, as well as in some eukaryotes and archaea $[13,14]$. Comparative genomic analysis has played a major role in the discovery and analysis of T-boxes $[9,15]$ and most riboswitches (reviewed in $[4,5]$ ). Several groups performed large-scale search for new RNA regulatory structures $[16,17]$. Analysis of RNA-based regulation often leads to non-trivial functional assignments for hypothetical genes and filling gaps in metabolic reconstruction (e.g. $[11,14,18,19])$.

Here we performed comparative analysis of candidate RNA regulatory elements in genomes of Actinobacteria. There are few known attenuators in these genomes. Those that have been experimentally studied are attenuators of the trp operons in Corynebacterium glutamicum [20] and Streptomyces venezuelae [21]. Studies of attenuator-like structures upstream of the ilvB and leuA genes of Streptomyces coelicolor produced somewhat ambivalent results. Indeed, although candidate leader peptides and alternative RNA structures were found upstream of the $i l v B$ and leuA genes, reminiscent of the classical attenuators, the mutation analysis demonstrated that the regulatory mechanism is not attenuation in the strict sense: mutations in candidate regulatory codons in the leader peptide of the $i l v B$ gene had no effect on regulation, and, although mutations in the leader peptide of leuA had some effect, it was not consistent with classical attenuation [22]. Computational analysis identified several types of riboswitches: THI-elements [14], RFN-elements [18], B12-elements [19], all of them regulating genes of cofactor metabolism by sequestering the Shine-Dalgarno box and start codon, and interfering with initiation of translation.

\section{Results and discussion}

Following an approach described previously [8], we systematically analysed the upstream regions of amino acid biosynthesis and aminoacyl-tRNA synthetase operons. Candidate regulatory structures were found upstream of genes involved in tryptophan, cysteine, and leucine metabolism. Candidate T-boxes were observed upstream of isoleucyl-tRNA synthetase genes. No conserved structures were observed upstream of genes from other amino acid biosynthesis pathways.

\section{Tryptophan}

The trp operons are preceded by classical candidate attenuators in all considered genomes of Corynebacterium spp. and Streptomyces spp. (Fig. 1). The leader peptides have double or triple repeats of regulatory UGG codons. All terminators are GC-rich and followed by poly-U-tracts. The antiterminator and terminator hairpins in all genomes contain complementary triples gGCC-rGCy-GGCC where absolutely conserved positions are set in capitals. This is analogous to the situation in proteobacteria, where the patterns involved in multiple interactions within attenuators are conserved at large evolutionary distances [8]. In $C$. diphteriae, candidate attenuators were found upstream of both biosynthetic operons $\operatorname{trp} B_{1} E D G C$ and $\operatorname{trp} B_{2} A$. A candidate attenuator was found upstream of the tryptophanyl-tRNA synthetase gene $\operatorname{trp} S_{2}$ in $S$. avermitilis.

\section{Cysteine}

The upstream regions of the cys operon in Mycobacterium spp. and Propionibacterium acnes and the cbs gene of Bifidobacterium longum contain short open reading frames encoding candidate leader peptides with runs of cysteine codons near the stop codon (Fig. 2a). The upstream regions of Mycobacterium spp. are very similar and can be aligned (Fig. 2b). However, they do not contain any conserved hairpins that could serve as terminators of transcription. One possibility is that this region contains rhodependent terminators similar to the situation in the tryptophanase operon tna of E. coli [23]. Indeed, Mycobacteium spp. have few rho-independent terminators $[24,25]$. On the other hand, all Mycobacterium genomes contain the components of the rho-dependent termination mechanism, rho, nusG, nusA, nusB. The region between the candidate leader peptide ORFs and the first genes in the $c y s$ operons contain polyY motifs that could serve as Rhobinding sites [26-28]. However, these motifs are not conserved, and thus this prediction is rather weak.

The cysteine operons in M. avium and M. leprae contain additional hypothetical genes, MAP2122 and ML0840 respectively, that are $62 \%$ identitical but have no other reliable homologs. 


\begin{tabular}{|c|c|c|c|c|}
\hline \multicolumn{5}{|l|}{ a) } \\
\hline Bacterium & Locus & Gene & Gene coordinates & Protein \\
\hline C. diphtheriae & NC_002935 & $\begin{array}{l}\operatorname{trpB} 1 \\
\operatorname{trpB2}\end{array}$ & $\begin{array}{l}2456701 . .2458032 \\
2465139 . .2466365\end{array}$ & $\begin{array}{l}\text { NP_940652 } \\
\text { NP_940660 }\end{array}$ \\
\hline C. efficiens & NC_004369 & $\operatorname{trp} E$ & $3052837 . .3054504$ & $N^{-}{ }_{-} 739478$ \\
\hline C. glutamicum & NC_003450 & $\operatorname{trp} E$ & $3233404 . .3234960$ & NP_602223 \\
\hline S. avermitilis & NC_003155 & $\begin{array}{l}\operatorname{trps} 2 \\
\operatorname{trpE} 1\end{array}$ & $\begin{array}{l}\text { complement }(5757496 . .5758491) \\
\text { complement(7320283..7322268) }\end{array}$ & $\begin{array}{l}\text { NP_825902 } \\
\text { NP_827260 }\end{array}$ \\
\hline coelicolor & NC_003888 & $\operatorname{trpE}$ & $2276703 . .2278607$ & NP_6263 \\
\hline
\end{tabular}

\section{b)}

Bacterium

C. diphtheriae

C. diphtheriae

C. efficiens

C. glutamicum

S. avermitilis

S. avermitilis

S. coelicolor

\section{c)}

Bacterium

C. diphtheriae

C. diphtheriae

C. efficiens

C. glutamicum

S. avermitilis

S. avermitilis

S. coelicolor

S. venezuelae

C. diphtheriae

C. diphtheriae

C. efficiens

C. glutamicum

S. avermitilis

S. avermitilis

S. coelicolor

S. venezuelae
Operon

trpB1EGDC1

$\operatorname{trpB2A}$

$\operatorname{trpEGDCBA}$

trpEGDCBA

trpS2

trpE1

trpE

Operon $\operatorname{trpB1EGDC1}$

$\operatorname{trpB2A}$

trpEGDCBA

$\operatorname{trp} E G D C B A$

trpS2

trpE1

trpE

trpE

\begin{tabular}{|c|c|c|}
\hline \multicolumn{3}{|c|}{ Leader peptide } \\
\hline 2456514 & - - - - - -MNAHNWWWRA - - - - - & 2456543 \\
\hline 464983 & - - - - -MNAAFKFWWRA - - - - - & 2465015 \\
\hline 3052621 & VNNFCQ & 3052671 \\
\hline 3233152 & VNNSCLS & 3233199 \\
\hline 5758647 & - - -MTTR & 575860 \\
\hline 7322414 & - - -MFAH & 732236 \\
\hline 2276540 & $---\mathrm{MFAH}$ & 227659 \\
\hline
\end{tabular}

Attenuator

uggugguggcgcgcuuaacc.gcgggec.guuuu. . .cacgcauucauuuc. uucugguggcgcgccuagcaggegggccccuuuugugugagcauucaccaca uggugguggcgcgcuagauaagegggcccacggaucaccaaguuguuuucac uggugguggcgcgcuaacuaagcgagccugacaccucaaguuguuuucacuu caguggugggccgccuga. cggeg.gccguacacacguauguacuc..... uggugguggaccgcucauccggeg.gcccacugacugcgcgu....... uggugguggaccgcucacccggcg.gcccacugacugcgcgcg...... uggugguggaccgcucacccggeg.gcccacugaucgcgcgu.......

\section{Figure I}

Leader peptides and candidate attenuators upstream the trp operons in Corynebacterium and Streptomyces spp. a) Coordinates and protein identifiers of the first genes in the operons. b) Alignment of the leader peptides. The numbers denote genome positions of the aligned fragments. c) Alignment of the attenuators. Tryptophan and stop codons are shown in bold. The terminator hairpins are highlighted in grey, the antiterminator hairpins are underlined. The alignment contains fragments between the tryptohan codons and the terminator hairpin followed by poly-U-tracts. The numbers denote genome positions of the aligned fragments. 


\begin{tabular}{|c|c|c|c|c|}
\hline Bacterium & Locus & Gene & Gene coordinates & Protein \\
\hline M. avium & NC_002944 & MAP2122 & $2351330 . .2352622$ & NP_961056 \\
\hline M. bovis & NC_002945 & cysk1 & $2586392 . .2587324$ & NP_856011 \\
\hline $\begin{array}{l}\text { M. tub } \\
\text { CDC1551 }\end{array}$ & NC_002755 & cysk & $2604640 . .2605572$ & NP_336875 \\
\hline M. tub H37Rv & NC_000962 & cysK & $2608794 . .2609726$ & NP_216850 \\
\hline M. leprae & NC_002677 & ML0840 & complement(997285..998589) & NP_301634 \\
\hline M. marinum & gnl|Sanger_216594|mar22d05.p1 & c cysk & complement(136548..137477) & (unfinished) \\
\hline$P$. acnes & NC_006085 & cysk & $1047389 . .1048324$ & YP_055674 \\
\hline B. longum & NC_004307 & cbs & $1006495 . .1007721$ & NP_696325 \\
\hline
\end{tabular}

b) Bacterium

M. avium

M. bovis

M. tub CDC1551

M. tub H37Rv

M. leprae

M. marinum

$P$. acnes

B. longum

c)

M. avium

M. bovis, tub

M. Ieprae

M. marinum

Operon
XcysKE
cysK1E
cysKE
cysKE
XcysKE
cysKE
cysK
cbs

Leader peptide

2351124 MQHRLQPRFAPSRCLVVACCCCCCR 2351177

2586122 MQQAIQLRFILPRRLAVGCCCC--- 2586187

2604371 MQQAIQLRFILPRRLAVGCCCC--- 2604436

2608526 MQQAIQLRFILPRRLAVGCCCC--- 2608591

0998791 MHQSTQPRFVFTRRFTVDCYCRCC- 0998742

0138059 MQQAAQLSFVLTRCPAVDCCCC--- 0137994

$1047061 \quad$ MTSAMMVCICRCCC- 1047102

1007876

MQIISCCCR - 1007850

\begin{abstract}
M. marinum
\end{abstract}

M. avium
$M$. bovis, tub
M. leprae
$M$. marinum

M. avium

M. bovis, tub

M. leprae

M. marinum

\begin{abstract}
RBS Start
uauaguggugacaugcaacaccgccuacagccgcgcuuu

uauagugggcccaugcaacaggccauacagcugcgcuuu

uauaguggaccuaugcaucaguccacacagccacgcuuu

uauaguagagccaugcaacaggccgcacagcugagcuuu
\end{abstract}

Cys tract

gсCCCgucgcgcugccuugucguggccuguuguugcuguuguugucgu auccucccgcgccgccucgccgugggcuguuguuguugu........ gucuuuacgcgccgcuuuaccguggacuguuauugucgcuguugc... . guccucacgcgcugccccgccguggacuguuguuguugcugu.....

stop and putative Rho binding site ugAUUUCCgcaaGCCCUCugacgcuguagaaAUCCCCgcgcucGCCCCUgCCCg ugAUUCCUg · gcguccacagcaAUUCCUCgCGCUCUUgcCcg ugAUUCCUgac . ACCUUUUaa cGCUCUCagcaaaucauuca cGUUCUCgccua ugAUUCCUgac.gcguucugaccguccaguaaucgucGCCUCUgucgccucaugg

Figure 2

Leader peptides upstream the cys operons in Mycobacterium spp. and $P$. acnes and cbs operon in B. longum. a) Coordinates and protein identifiers of the first genes in the operons. b) Alignment of the leader peptides. The numbers denote genome positions of the aligned fragments. c) DNA alignment of the leader peptide genes. Start, cysteine and stop codons are shown in bold; candidate Rho-binding sites are shown in capitals.

\section{Leucine}

The upstream regions of the $i l v B$ genes (operons ilvBNC, ilvBHC, ilvBser $A_{1}$ ) in Corynebactecterium, Mycobacterium, Streptomyces species contain short ORFs with runs of isoleucine, valine and leucine codons overlapping the candi- date terminator hairpins followed by polyU-runs (Fig. 3). However, the exact mode of regulation is not clear, as experimental substitution of possible regulatory codons upstream of the ilvBNC operon in S. coelicolor had no effect on regulation or expression of $i l v B$ [23]. 


\begin{tabular}{|c|c|c|c|c|}
\hline \\
\hline Bacterium & Locus & Gene & Gene coordinate & Protein \\
\hline C. diphtheriae & NC_002935 & $i l v B$ & $1082013 . .1083971$ & NP_939459 \\
\hline C. efficiens & NC_004369 & ilv $B$ & $1432330 . .1434327$ & NP_737975 \\
\hline C. glutamicum & NC_003450 & ilvB & $1338131 . .1340011$ & NP_600493 \\
\hline M. tuberculosis H37Rv & NC_000962 & ilvB & complement(3361127..3362983) & NP_217519 \\
\hline M. tuberculosis CDC1551 & NC_002755 & ilvB & complement(3355506..3357362) & NP_337598 \\
\hline M. bovis & NC_002945 & ilvB1 & complement(3317745..3319601) & NP_856673 \\
\hline M. leprae & NC_002677 & $i l v B$ & complement(2044335..2046212) & NP_302166 \\
\hline M. avium & NC_002944 & ilvB1 & complement(3379032..3380900) & NP_961972 \\
\hline M. marinum gnl|s & $6594 \mid \operatorname{mar} 755 \mathrm{~h}$ & 1.p2k1114 & complement(164709..166565) & (unfinished) \\
\hline S. avermitilis & NC_003155 & ilvB & complement(3354433..3356283) & NP_823909 \\
\hline S. coelicolor & NC_003888 & ilv $B$ & $6003117 . .6004958$ & NP_629647 \\
\hline
\end{tabular}

\section{b)}
Bacterium
C. diphtheriae
C. efficiens
C. glutamicum
M. tuberculosis H37Rv
M. tuberculosis CDC1551
M. bovis
M. leprae
M. avium
M. marinum
S. avermitilis
S. coelicolor

\section{c)}

Bacterium

C. diphtheriae

C. efficiens

C. glutamicum

M. tuberculosis

$M$. bovis

M. leprae

M. avium

M. marinum

$S$. avermitilis

S. coelicolor

Operon
ilvBHC
ilvBHC
ilvBHC
ilvBNC
ilvBNC
ilvB1NC
ilvBNC
ilvB1N
ilvB
ilvBNC
ilVBNC

$\begin{array}{rrr} & \text { Leader Peptide } \\ 1081747 & \text { MNIIRLVVITTRRLP } & 1081791 \\ 1432212 & \text { MTSIRPVVIVAARRLP - } & 1432259 \\ 1337840 & \text { MTIIRLVVVTARRLP } & 1337884 \\ 3363152 & \text { MDKAGKPGMLVVIGRRVGA } & 3363096 \\ 3357528 & \text { MDKAGKPGMLVVIGRRVGA } & 3357472 \\ 3319767 & \text { MDKAGKPGMLVVIGRRVGA } & 3319711 \\ 2046378 & \text { MLVVICQRVGG } & 2046346 \\ 3381051 & \text { MLVVI-RRVGA } & 3381022 \\ 166742 & \text { MDTAGTPGKLVVLGRRVVA } & 166686 \\ 3356481 & \text { MRTRILVLGKRVG } & 3356443 \\ 6002909 & \text { MRTRILVLGKRVG } & 6002947\end{array}$

Figure 3

Candidate leader peptides and terminators upstream the ilv opreron in Actinobacteria. a) Coordinates and protein identifiers of the first genes in the operons. b) Alignment of the leader peptides. The numbers denote genome positions of the aligned fragments. c) Alignment of the terminators. The terminator hairpins are highlighted in grey.

Classical candidate attenuators were found upstream of leuS (leucyl-tRNA-synthetase) in S. avermitilis and S. coelicolor. Each of them contains an ORFs encoding the leader peptide, as well as the antiterminator and terminator hairpins (Fig. 4).
Sequences upstream of the isopropylmalate synthase genes leuA contain a number of candidate regulatory sequences, together named the LEU element (Fig. 5, 6). Firstly, there is an upstream ORF encoding a candidate leader peptide with a run of leucine codons (Fig. 7). 

a)
Bacterium
S. avermitilis
Locus
Gene
Gene coordinates
leuS 6661895..6664783
S. coelicolor
NC 003155
NC_003888
leus
Protein
NP 826665
NP_626809

b)
$\begin{array}{llllllllllll}M & R & A & V & R & L & L & L & S & E & P & R\end{array}$
S. avermitilis 6661741 augcgugccguacgccuucugcuuagcgagccgcgcugaucagcccagaccacugacga
S. coelicolor 2778624 augcgugccguacgccuucugcuuagcgagccgcgcugaucagucccgaccccggucgu
S. avermitilis ..uuc.guggucggaaucggcgcggcguccccuccugugcgagggguuuuuucauu 6661852
S. coelicolor aguccgguggccggaaucggcgcggcguccccuccugugcgaggggauuuucauu 2778510

\section{Figure 4}

Candidate attenuators upstream the leuS opreron in Streptomyces spp. a) Coordinates and protein identifiers of the leuS genes. b) Alignment of the attenuators. Start, leucine and stop codons are shown in bold. The terminator hairpins are highlighted in grey, the antiterminator hairpins are underlined. The alignment contains fragments between the leader peptide ORFs and the terminator hairpin followed by poly-U-tracts.

Secondly, this region may fold into a pseudoknot with an additional stem at its base formed by pairing of the leucine codon run with the Shine-Dalgarno box of the leuA gene (Fig. 5, 8). Finally, the same region may form an alternative hairpin with the same base stem (Fig. 6).

A similar pseudoknot was found in B. longum within a gene encoding a transposase. The latter is homologous to the IS1554 transposase of $M$. tuberculosis and M. bovis (66\% identity), a putative transposase in C. efficiens $(40 \%$ identity), putative IS256 family transposases of S. avermitilis (31\% identity), hypothetical protein MAP2274 of $M$. avium (29\% identity), and some other putative transposases from B. longum, C. efficiens, M. tuberculosis, $M$. bovis, R. xylanophilus, S. avermitilis, S. coelicolor (Fig. 9a). However, only the $B$. longum transposase contains a fragment that may fold into the pseudoknot (Fig. 9b), whereas other transposases, although highly similar on the protein level in the corresponding region, contain a number of non-complementary mismatches in synonymous codon positions and thus have lost the pseudoknot folding potential.

\section{T-boxes}

Candidate T-box structures were found upstream of the ileS genes from several Actinobacteria. They are unusual, as instead of terminators, they contain hairpins sequestering the Shine-Dalgarno boxes of the ileS genes (Fig. 10).
Thus it is likely that the regulatory mechanism involves inhibition of translation initiation. To our knowledge, this is the first example of a T-box acting on the level of translation.

\section{Conclusion}

Candidate regulatory elements were found upstream of genes involved in the tryptophan, cysteine and branched chain amino acids metabolism. No conserved RNA regulatory structures were observed upstream of histidine, threonine, phenylalanine, tyrosine, arginine, lysine, methionine operons, although orthologous genes involved in the latter pathways are regulated on the RNA level in other species: methionine and lysine by the S-box and L-box riboswitches respectively [3-5], histidine, threonine and phenylalanine by attenuators $[7,8]$, tyrosine and arginine by T-boxes [12].

Attenuators of the classical type were observed upstream of the aminoacyl-tRNA-synthetase genes $\operatorname{trpS}$ and leuS in some Streptomyces genomes, similar to those observed in gamma-proteobacteria, (e.g. the pheST operon) [7]. In contrast, in Firmicutes, most aminoacyl-tRNA-synthetase genes are regulated by tRNA-dependent antitermination (T-boxes) and none by classical attenuation $[2,9,15]$. No classical T-boxes were found in Actinobacteria, but unusual T-boxes, possibly regulating initiation of translation, 
C. diphtheria

C. efficiens

C. glutamicum

$K$. radiotolerans

M. avium

M. bovis

M. Ieprae

M. marinum

M. smegmatis

S. avermitilis

S. coelicolor

T. fusca

L. XYli

N. farcinica

A. naeslundii

B. Iongum cuucuccuucuu...... egcegeggegggucacaggeuuaacgucceuua gcucuucuucuu...... cgecgeggegggucccagaggucaua..... cuacuucuucuu...... cgccgeggegggucccagaggucuua...... a accuccuccuuc. . gucgecgeggeggggecag . . . . . . . . . . . cgggugcuccuccucggacgecgegacggggucugauu. . . . . . . . . . .

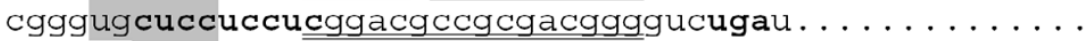
cagguacuccuccucgaacgccgegacggggucugau...........

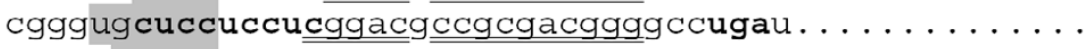
cgggugcuccuucucggacgccgeggcggg. . . . . . gucuga . . . . . . gggcugcuccuccuuagcugcegcggegagggccuguaag ......... . gggcugcuucuccuuagcugccgcggcgagggccuguag . . . . . . . . . . . . . .

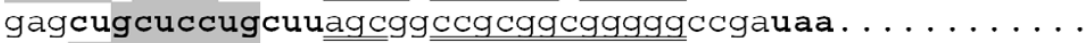

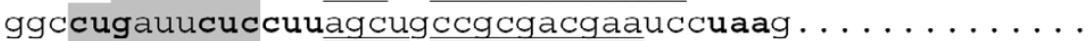
cgggcucuucuucucggecgecgegacgggqucugau........... gugagccuccugcuuagucgccgcggcggggccuga.......... ggcguggaucuggagggcggccgcgacgug . . cugggc . . . . . . . .

Cd cacacagccggcuc. cccgucgcggaguucu........aguguagccggcug. . . . . . .

$\mathrm{Ce} . . \mathrm{gcgaccggca}$. cccgucgcggaguuu.......guguugccggucgugaacccg

$\mathrm{Cg}$. cacgaccggcau. cccgucgcggaguuu........gguguugccggucgug.....

$\mathrm{Kr} \ldots$ cuaggecggucucecegucgegggaccucguc ...gugcg. $\operatorname{cgccggec} \ldots \ldots \ldots$

$\mathrm{Ma}$. ccagaccggcuu. cccgucgcgggu.guucgc ....gaug. cgccggucug.......

$\mathrm{Mb}$. ccagaccggcuu. cccgucgcgggacguucgc ....gaug. cgccggucug.......

M1 . cccagaccggcug. cccguuguggaa.guucac....uaug.cgccggucug......

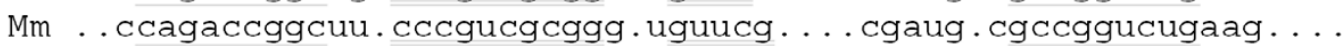

Ms . .ucagaccggcuu. cccgucgcggg.uguuu . . cgcgaug. cgccggucga . . . . . .

$\mathrm{Sa}$. cagaggccgacccccuccccgcgg . . agucugg. cguugcgccgucggccg.......

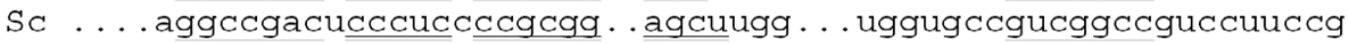

Tf ...ggggccggcucccucgecgcggagguucgac.cugucugcugucggccg.......

Lx uuccgggcc...uccuucgucgcgg. aguucguc.........guuggcucuccc....

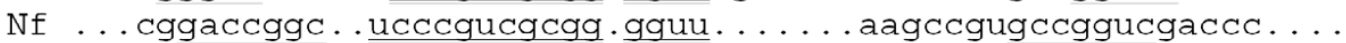

An ...caggccggcaccccgaccgcggcugacucgu......ccugcucggccacguucgcg

Bl aucugggc..gucg.cccgccgcggagggcgcacgcuauuggcugucggugcucac. .

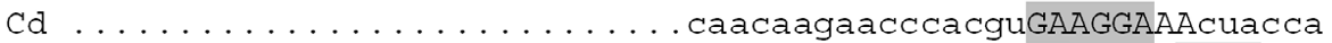

Ce caacagcgcuagaguuugauuccagaaaacaagcgcacacuccacGAAAGAUGagcacccauc

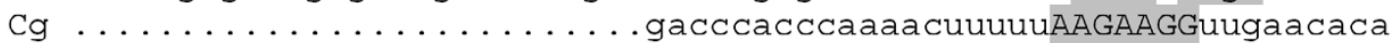

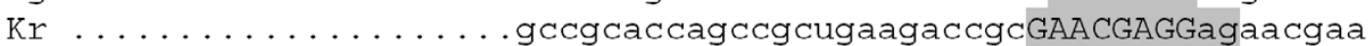

$\mathrm{Ma} \ldots \ldots \ldots \ldots \ldots \ldots$ aggunccuucugauaucCCCGGAGCAAuca cC

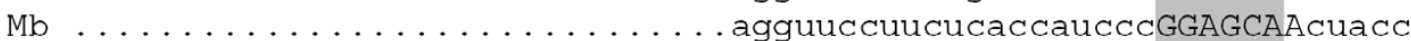

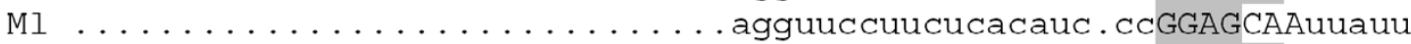

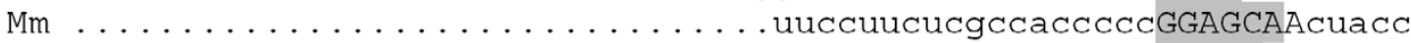

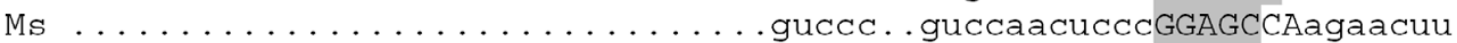

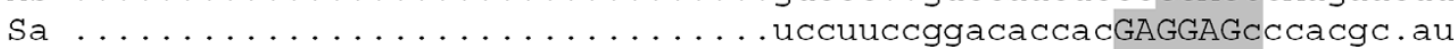

Sc .... gacacgcggacgacgcggacaccgccgagauccgcggacaucacGAGGAGcccacgccau

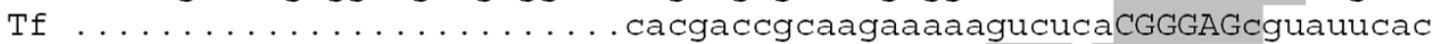

$\mathrm{Lx} \ldots \ldots \ldots \ldots$. . . . . . . . . . . .

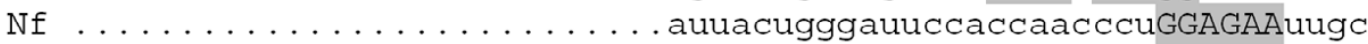

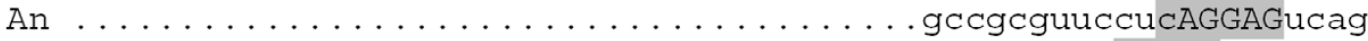

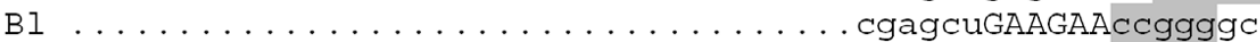

\section{Figure 5}

Alignment and RNA secondary structures of the leuA upstream regions (LEU elements). The stem at the base is highlighted in grey, helices forming the pseudoknot are underlined and double underlined, leucine and stop codons are set in bold, the candidate Shine-Dalgarno boxes of the leuA are set in capitals. The last sequence is that of the transposase from $B$. longum (see the text). Sequences for M. bovis (Mb) and M. tuberculosis spp. (Mt and Rv) coincide. 
C. diphtheria

c. efficiens

C. glutamicum

$K$. radiotolerans

M. avium

M. bovis

M. Ieprae

M. marinum

M. smegmatis

$S$. avermitilis

S. coelicolor

T. fusca

L. xyli

N. farcinica

A. naeslundii

$B$. Iongum cuucuccuucuu......cgecgeggegggucacaggcuuaacgucccuua gcucuucuucuu......cgccgcggcgggucccagaggucauaa..... cuacuucuucuu. . . . cgccgeggegggucccagaggucuuaa..... aaccuccuccuuc. . gucgecgcggcggggccag. . . . . . . . cgggugcuccuccucggacgccgcgacggggucugauu........ cgggugcuccuccucggacgecgegacggggucugau......... cagguacuccuccucgaacgecgegacggggucugau......... cgggugcuccuccucggacgccgcgacggggccugau......... cgggugcuccuucucggacgecgeggeggg. . . . . gucuga . . . . . gggcugcuccuccuuagcugccgcggcgagggccuguaag ........ gggcugcuucuccuuagcugccgcggcgagggccuguag . . . . . . . gagcugcuccugcuuageggecgeggegggggecgauaa......... ggccugauucuccuuagcugccgcgacgaauccuaag.......... cgggcucuucuucucggecgecgegacggggucugau ......... gugagccuccugcuuagucgecgeggeggggecuga......... ggcguggaucuggagggcggccgcgacgug. . cugggc..........

Cd cacacagccggcuc. cccgucgcggaguucu........aguguagccggcug....... . . .

$\mathrm{Ce} . . \mathrm{gcgaccggcac}$. cccgucgcggaguuu........guguugccggucgugaacccg

$\mathrm{Cg}$. .cacgaccggcau. cccgucgcggaguuu.......gguguugccggucgug ..... .

$\mathrm{Kr}$.. cuaggccggucuccccgucgcgggaccucguc...gugcg.cgccggcc........

$\mathrm{Ma} \mathrm{..ccagaccggcuu.cccgucgcgggu.guucgc} \mathrm{....gaug.cgccggucug} \mathrm{......}$

$\mathrm{Mb}$. ccagaccggcuu.cccgucgcgggacguucgc ....gaug.cgccggucug . . . . . .

M1 . cccagaccggcug. cccguuguggaa.guncacu. ....aug. cgccggucug. ......

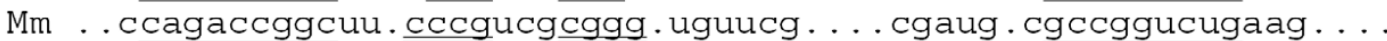

Ms . .ucagaccggcuu. cccqucgcggg.uguuu . . cgcgaug. cgccggucga . . . . . .

$\mathrm{Sa}$. cagaggcegacccccuccccgcggag. .ucugg. cguugcgccgucggccg.......

Sc ..... aggccgacucccuccccgcggag. . cuugg ...uggugccgucggccguccuuccg

Tf ...ggggccggcucceucgccgeggagguucgac.cugucugcugucggccg.......

Lx uuccgggcc...uccuucgucgeggag.uucguc........ guuggcucuccc....

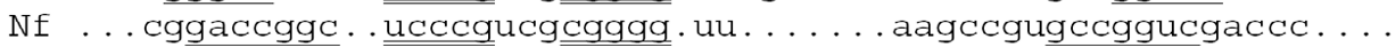

An ...caggccggcaccccgaccgcggcugacucgu . . . . . ccugcucggccacguucgcg

Bl aucugggc..gucg. CcCgccgeggagggcgcacgcuauuggcugucggugcucac. .

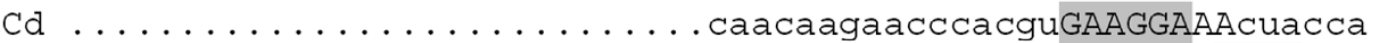

Ce caacagcgcuagaguuugauuccagaaaacaagcgcacacuccacGAAAGAUGagcacccauc

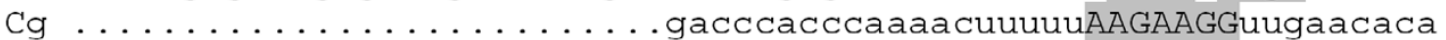

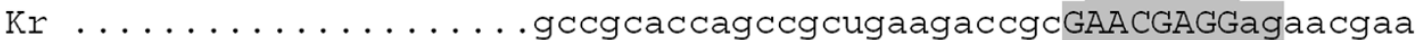

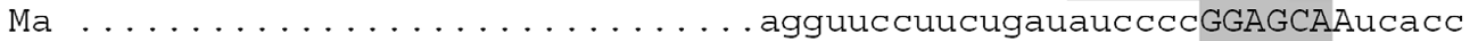

$\mathrm{Mb} \ldots \ldots \ldots \ldots \ldots \ldots \ldots \ldots \ldots \ldots \ldots \ldots \ldots \ldots$ agguccuucucaccaucccGGAGCAAcua cc

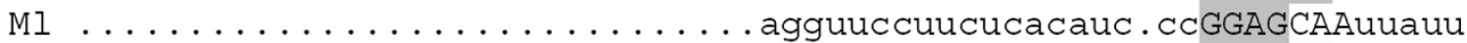

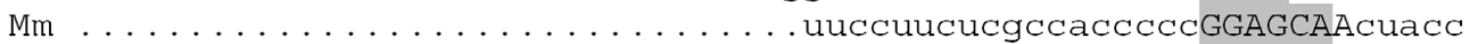

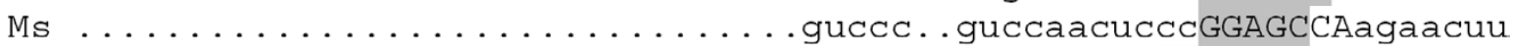

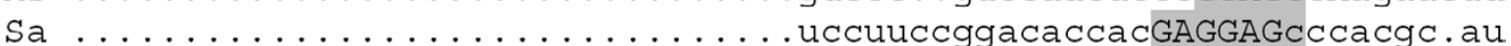

$\mathrm{Sc}$.... gacacgcggacgacgcggacaccgccgagauccgcggacaucacGAGGAGcccacgccau

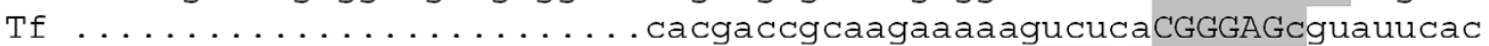

$\mathrm{Lx} \ldots \ldots \ldots \ldots \ldots \ldots$ gacagaccgcGAAGAGAuaucggacC

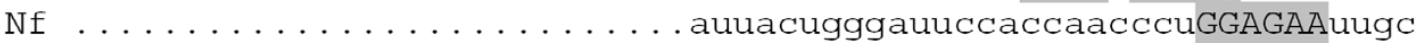

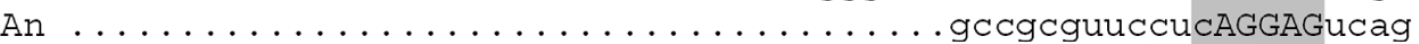

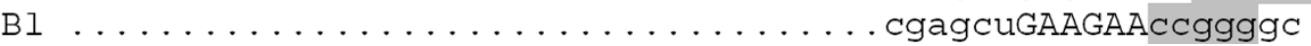

Figure 6

Alternative RNA secondary structure in LEU elements. The stem at the base is highlighted in grey, two internal helices are underlined and double underlined, other notation as in Fig 5. 

a)

Bacterium
C. diphtheria
C. efficiens
C. glutamicum
K. radiotolerans
M. avium
M. bovis
M. tub CDC1551
M. tub H37Rv
M. leprae
M. marinum
M. smegmatis
S. avermitilis
S. coelicolor
T. fusca
Locus
NC_002935
NC_004369
NC 003450
AAËF02000060
NC_002944
NC_002945
NC_002755
NC_000962
NC_002677
gnl|Sanger_216594|mar428a07.p1k
Gene
leuA
leuA
Gene coordinates
complement(228555..230372)
complement(233589..235439)
(adding 105 nucleotides)
leuA complement(266151..268001)
leuA
leuA
leuA
leuA
leuA
complement(3238..4965)
333789..335633
4091088..4093193
4145949..4147928
$4153737 . .4155671$
$2754640 . .2756463$
192528..194345
gnl|TIGR_246196|contig:3563:m_smegmatis 6334690..6336495
NC 003155
NC_003888
NZ_AAAQ02000002
leuA2
leuA
6774328..6776049
leuA
complement(2725480..2727201)
349237..350943
(adding 27 nucleotides)
NC_006087
leuA
complement(1501628..1503400)
leuA
complement(322994..324787)
gnI|TIGR_240017|contig:1063:a_naeslundii $594374 . .596211$ (unfinished)
Protein
NP_938656
NP_736826
NP 599502
EAM̄73829
NP_959246
NP 857375
NP_338367
NP_218227
NP_302512
(unfinished)
(unfinished)
NP 826778
NP_733575
ZP_00293601
YP_062368
YP-116514

b)

Bacterium

C. diphtheria

C. efficiens

C. glutamicum

K. radiotolerans

$M$. avium

$M$. bovis

M. tub CDC1551

M. tub H37Rv

$M$. leprae

M. marinum

M. smegmatis

$S$. avermitilis

S. coelicolor

T. fusca

L. xyli

N. farcinica

A. naeslundii

\section{6}

235612

268124

5097

333705

4090959

4145866

4153611

2754521

192399

6334564

6774199

2727361

349104

1503533

324906

594266
Leader peptide

MNRANLLLLRRGGSQA- 230459

MFSSHERSALLLRRGGSQRS 235553

MTSRANLLLLRRGGSQRS 268095

VARLENLLLRRRGGAS - $\quad 5050$

VADVQRVLLLGRRDGV-- 333752

VLHVQRVLLLGRRDGV - 4091006

VLHVQRVLLLGRRDGV - 4145913

VLHVQRVLLLGRRDGV - - 4153658

VQQVLLLERRDGV - 2754559

VLCVQRVLLLGRRDG - - - 192443

VLGVQRVLLLGRRGGV- - 6334611

MRFGLLLLSCRGEGL - 6774243

MRFGLLLLSCRGEGL -2727317

MLRELLLLSGRGGGR - 349148

MRVTLGLVYGLILLSCRDES - - 1503474

MQRALLLGRRDGV- - 324868

VSLLLSRRGGA-- 594298

Figure 7

Candidate leader peptides in the LEU elements.

were observed upstream of the ileS genes in several genomes.

Despite the presense of conserved leader peptides upstream of some cysteine and leucine operons, the mode of regulation is unknown, as other attenuator elements are missing. One possible explanation is that attenuation of the cys operons in Mycobacterium spp. and P. acnes and the $c b$ s operon in B. longum involves Rho-dependent termination, similar to the tna operon of E. coli $[23,29]$. 


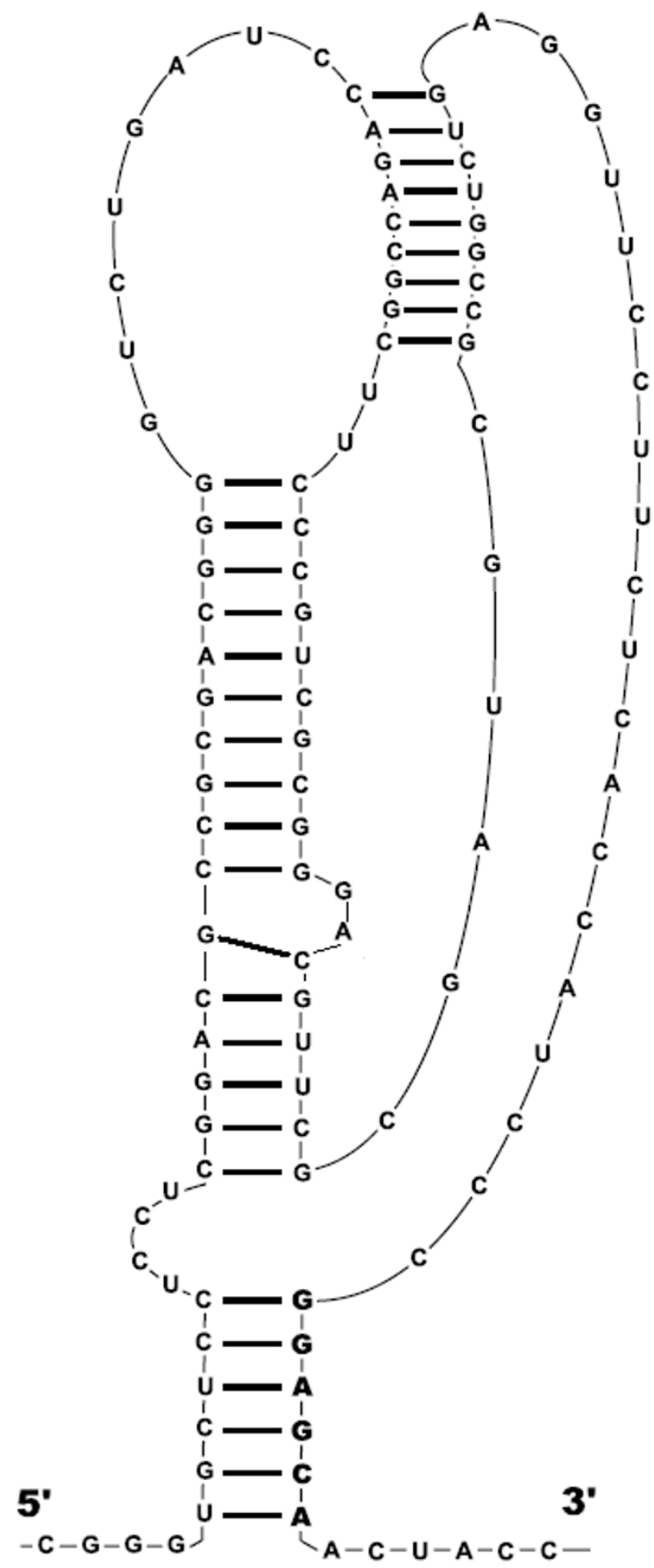

Figure 8

Candidate RNA pseudoknot upstream of the leuA operon in $\mathbf{M}$. bovis. The corresponding alignment is given Fig. 5. Boldface: the candidate Shine-Dalgarno box.
The most interesting case seems to be that of the leuA genes. The upstream regions of these genes contain several conserved elements (referred to as the LEU element) that can be interpreted in different ways. There are some architectural similarities with riboswitches, in particular, a compact structure with a stem at the base [5,30,31]. The latter is formed by interaction of a run of leucine codons and the Shine-Dalgarno box. Indeed, Actinobacteria seem to be the only taxonomic group where the base stems of riboswitches directly overlap the translation initiation site, without additional regulatory hairpins [5]. However, the LEU element differs from all known riboswitches, as the alignment of LEU elements does not contain conserved unpaired nucleotides that would be involved in tertiary interactions and form the ligand-binding pocket, as in the purine riboswitches whose spatial structure has been resolved [30,31] and in other riboswitches [5]. Thus direct binding of a small molecule to LEU elements seems unlikely. On the other hand, there is experimental evidence that mutations in the leucine codons do not influence the regulation [22] and thus classical attenuation involving translation of a leader peptide also is an unlikely mechanism of regulation.

The above considerations make it likely that the LEU element is a binding site of some regulatory protein. To test for this possibility, we compared the pattern of phylogenetic distribution of LEU elements to phylogenetic distributions of all actinobacterial genes. The closest phylogenetic pattern was observed for orthologs of ML1624 from M. leprae: homologs of this protein with Evalues $<10^{-170}$ were found in all genomes containing LEU elements, but not outside Actinobacteria. The only unexplained fact is the presence of a homolog with the E-value $\sim 10^{-108}$ in $P$. acnes, which does not have a LEU element. The structure of the ML1642 protein is consistent with an RNA-binding regulatory role, as the protein contains an $\mathrm{N}$-terminal DEAD-box helicase domain (ProFam family PF00270, E-value $3.6 \cdot 10^{-6}$ ) that may be involved in unwinding of nucleic acids.

An additional enigma is the presence of a LEU elementlike sequence within a transposase gene. On the other hand, it may be a clue to the origin of LEU elements. One possibility is that the $B$. longum transposase represents an ancestral state where the LEU element was involved in maintenance or regulation of transposition. Situations when a regulatory site occurs within a regulatory and/or regulated gene are not very common, but they happen in mobile elements [32]. Other transposase genes may have lost the ability to form this structure due to mutations; notably, the protein sequence has not changed much (Fig. 9 ), as most mutations occurred in synonymous codon positions. A plausible scenario is that the transposase gene was inserted upstream of the leuA gene in the ancestral 
a)

$\begin{array}{lll}\text { Bacterium } & \text { Locus } & \text { Coordinates } \\ \text { B. longum } & \text { NC_004307 } & \text { 2124903.. 2126108 } \\ \text { M. bovis } & \text { NC_002945 } & \text { complement(1025963..1027282) } \\ \begin{array}{l}\text { M. tuberculosis CDC1551 } \\ \text { C. efficiens }\end{array} & \text { NC_002755 } & \text { complement(1025510..1026829) } \\ & \text { NC_004369 } & \text { complement(1561522..1562694) }\end{array}$

Protein

NC_004307

NP 854601

NP_335380

NP_738106

b)

\author{
B. longum \\ M. bovis \\ M. tuberculosis CDC1551 \\ C. efficiens
}

MDAAQVIEPAHAGQDVDEAAVAA MDAAQVIEPAHAGQDVDEAAVAA

MAKEKGLDLTGPDGLLKQFTKSVLETALDEEMTEHLGR * *AKHKKSKDGRAANTRNGTTAKTVVTDSVGPVGIEVPRDRDGS RELSGAERALVGDLVROARAEGVALTGPDGLLKALTKTVLEAALOEEMTEHLGY * * * DRHAAAGRGSGNSRNGSRNKKVITDACGOVEIAVPRDRNGT RELSGAERALVGDLVRQARAEGVALTGPDGLLKALTKTVLEAALQEEMTEHLGY * * * DRHAAAGRGSGNSRNGSRNKKVITDACGQVEIAVPRDRNGT MNAEMDAHLGYGHSDRDGKTAAGQGNHRNGYYPK RVDSNYGPIDVAVPRDRNGS

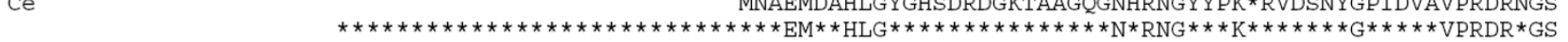

B1 FEPVVVRKRQRRLPGVDEVVLSLYARGLTTGEISAHFQEIYGADVSRETVSRITERVVAEKDEWCSRPLDRVYAAVFIDATVVKVRDG *QVANRAFYVAV $\mathrm{Mb}$ FEPVIVGKRKRRVTDVDRVVLSLYAKGLTTGEIAAHFADVYGVSVSKDTISRITDRVIEEMOAWWSRPLEKVYAAVFIDAIMVKIRDG *QVRNRPVYAAI Mt FEPVIVGKRKRRVTDVDRVVLSLYAKGLTTGEIAAHFADVYGVSVSKDTISRITDRVIEEMOAWWSRPLEKVYAAVFIDAIMVKIRDG *OVRNRPVYAAI Ce FLPTMVPKGSRRLTDVDDMI I * LYAGGMTVRDIQHHMITSMGVDISHETISAITDAVLDEVMIWQNRQLDDFYPVIFLDALRI KVRDGGRVVNKSVYLAI

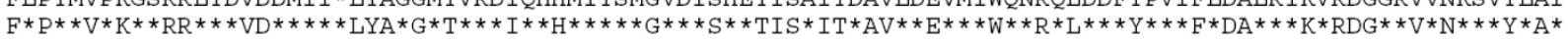

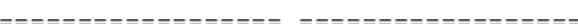

B1 GVDLEGGRDVLGIWASPA *AEGARYWLSVLTELKNRGVDDVFFLICDGLKGLPDAVGAVWPLAIVQTCVVHLLRNTFRYASKKDWDAIKRDVKPIYTAPS $\mathrm{Mb}$ GVDLDGHKDILGMWAGEGDGESAKFWLAVLTELRNRGVKDI FFLVCDGLKGLPDSVSAAFPLATVQTCI IHLIRNTFRYASRKYWDKISVDLKPIYTAAS Mt GVDLDGHKDILGMWAGEGDGESAKFWLAVLTDLRNRGVKDIFFLVCDGLKGLPDSVSAAFPLATVQTCI IHLIRNTFRYASRKYWDKISVDLKPIYTAAS Ce GVDIDGIKHILGIWLAKE **EGASFWANVCANLATRGVQDVFIVCCDGLKGLPQAVEATWPDSMVQTCVVHLIRAANRWVAYGDRKAVSAQLRKIYTAPT

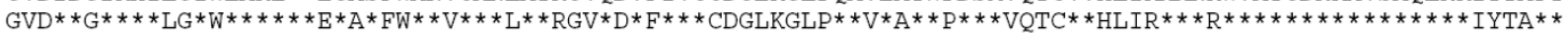

B1 **AAAAAAARDAMLDKWEARYPAIRRLWMDAWERFI PFLDYDVEIRRVICTTNAIESLNARFKRS IRARGHFPDEQAALKCMYLTVRSLDPTGKGRIRWS $\mathrm{Mb} * *$ AAEARLRYEEFAEKWGKPYPAITRLWDSAWEEFIPFLDYDVEIRRVPCSTNAIESLNARYRRAVRARGHFPNEQSALKTLYLVTRSLDPKGTGQTKWA Mt **AAEARLRYEEFAEKWGKPYPAITRLWDSAWEEFIPFLDYDVEIRRVPCSTNAIESLNARYRRAVRARGHFPNEQSALKTLYLVTRSLDPKGTGQTKWA Ce EDTAIAALEEFEASELGVK*YPQSAKVWRDAWDRFIPFLQFPPMARKVIYTTNSIESMNNELRKATRNRVQFTNDESAI KTLWLMICNIEDKRAAKRAKQ

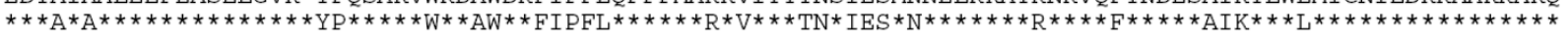

B1 ARWKPALNAFAITFADRWPSEGTQO VRWKPALNALAITFADRMPAAEER VRWKPALNALAITFADRMPAAEER GKRVAASSGRLIEGRKVANWKQAINQMAVAFPDRFEAYL $\star * * * * * * * * * * I * * * * * \star * * * * * * *$

c)

B. longum

M. bovis

M. tuberculosis

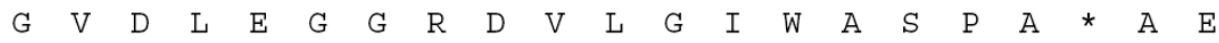

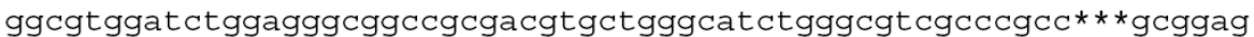

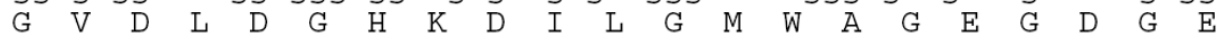
ggcgtcgacctcgacggccacaaggacatcctggggatgtgggccggcgaaggcgacggtgag ggcgtcgacctcgacggccacaaggacatcctggggatgtgggccggcgaaggcgacggtgag

B. longum

M. bovis

$\begin{array}{lllllllllllllllll}G & A & R & Y & W & L & S & V & L & T & E & L & K & N & R & G\end{array}$ ggcgcacgctattggctgtcggtgctcaccgagctgaagaaccggggc

M. tuberculosis

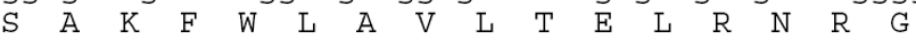
tcagccaaattttggctggcagtgctcaccgaactgcgcaatcgtggg $\begin{array}{llllllllllllllll}S & A & K & F & W & L & A & V & L & T & D & L & R & N & R & G\end{array}$ tcagccaaattttggctggcagtgctcaccgacctgcgcaatcgtggg

\section{Figure 9}

Multiple alignments of transposases. a) Coordinates and protein identifiers of putative transposases. b) Protein alignment. The fragment marked by the double line above corresponds to the $B$. longum fragment homologous to candidate pseudoknot and shown in the last line of Fig. 5. c) Nucleotide alignment of the region shown by the double line in (b). 
a)

\begin{tabular}{|c|c|c|}
\hline Bacterium & Locus & The ileS gene coordinates \\
\hline A. naeslundii & \multicolumn{2}{|c|}{ gnI|TIGR_240017|contig:1063:a_naeslundii complement(1311947. } \\
\hline C. diphtheriae & NC_0029335 & complement(1617227..1620385) \\
\hline C. efficiens & NC_004369 & $\begin{array}{l}\text { complement( } 2160737 . .2164195) \\
49 \text { codons removed }\end{array}$ \\
\hline C. glutamicum & NC_003450 & complement(2270986..2274150) \\
\hline M. avium & NC_002944 & $1324371 . .1327532$ \\
\hline M. bovis & NC_002945 & $1720532 . .1723657$ \\
\hline M. tub H37Rv & $\mathrm{NC}^{-} 000962$ & $1736519 . .1739644$ \\
\hline M. tub CDC1551 & NC_002755 & $1736672 . .1739797$ \\
\hline M. marinum & gnl|Sanger_216594| & $88 \mathrm{e} 12 . \mathrm{s} 1 \mathrm{k}$ complement(184205..187372) \\
\hline M. leprae & NC_00267̄ & $1410785 . .1413964$ \\
\hline N. farcinica & NC_006361 & $1932119 . .1935247$ \\
\hline$P$. acnes & NC_006085 & $268050 . .271394$ \\
\hline R. xylanophilus & NZ_AAEB01000029 & complement(26358..29492) \\
\hline S. avermitilis & $N C^{-0} 03155$ & complement(7371348..7374491) \\
\hline S. coelicolor & NC_003888 & $2227237 . .2230380$ \\
\hline T. fusca & NZ_AAAQ02000011 & complement(75752..78934) \\
\hline
\end{tabular}

Protein unfinished NP_939931 NP_738653

NP_601350 NP_960180 NP_855215 NP 216052 NP_336040 unfinished NP_301871 YP_-117986 YP_054935 ZP_00187197 NP_827306 NP_626335 ZP_00291779

b)

An 1315386 cCgucccggauggggcgcgcaguacggcaagcgAGGUGGUACCGCGgugcggcaccagccgggcaccagccCcggu

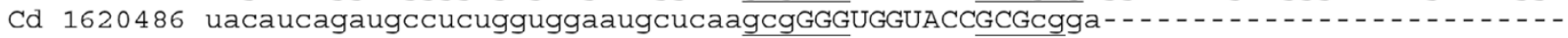

Ce 2164019 -gguggccuguuggugggCCgcagguucaagcaGGGUGGUACCGCGuccggauca - . . . . . . . . . . . . Cg 2274270 aacgaaguggagcuaguuaauuuagcucaagcuGGGUGGUACCGCGuCCguuu- . . . . . . . . . . . . . . . . -

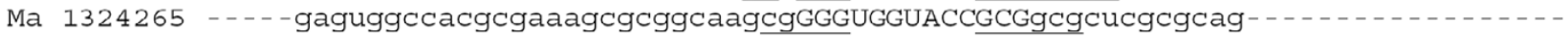

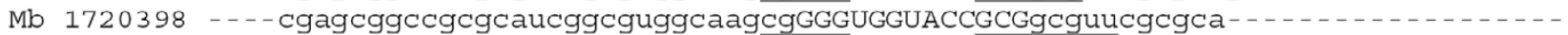

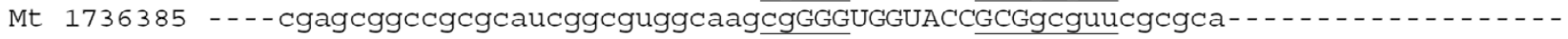
Mt 1736538 ----cgagcggCCgCgCaucggcguggcaagCgGGGUGGUACCGCGgCguuCgCgCa- - - - - - - - - - - - - M1 1410679 - - --aguggCCgugcguucgcgugCggcaagcgGGGUGGUACCGCGgCgCuCgCgCac - . Mm 187479 aaauugagCggCCgCacucaggugCggcaagCgGGGUGGUACCGCGgCgCuCgCgCa - . $\ldots$

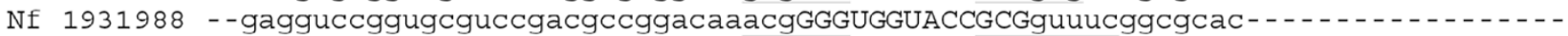

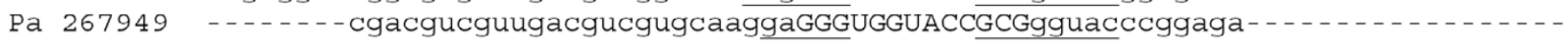
RX 29622 agcgguccggggcCgcgaggccucgggcaagcaGGGUGGUACCGCGagagcCgcuucuuuggagaaaga- - - - - - Sa 7374620 ---ggugcacacagggcgccggggagccaaggaGGGUGGUACCGCGggagcgcgccgcacacggcguacggaaaga Sc 2227135 -.-gagcacacgacgcaccggcCgggccaaggaGGGUGGUACCGCGggagca - . . . . . . . . . . . . . . . Tf $79034 \ldots \ldots-$ $\mathrm{T}-\mathrm{b} o \mathrm{x}$

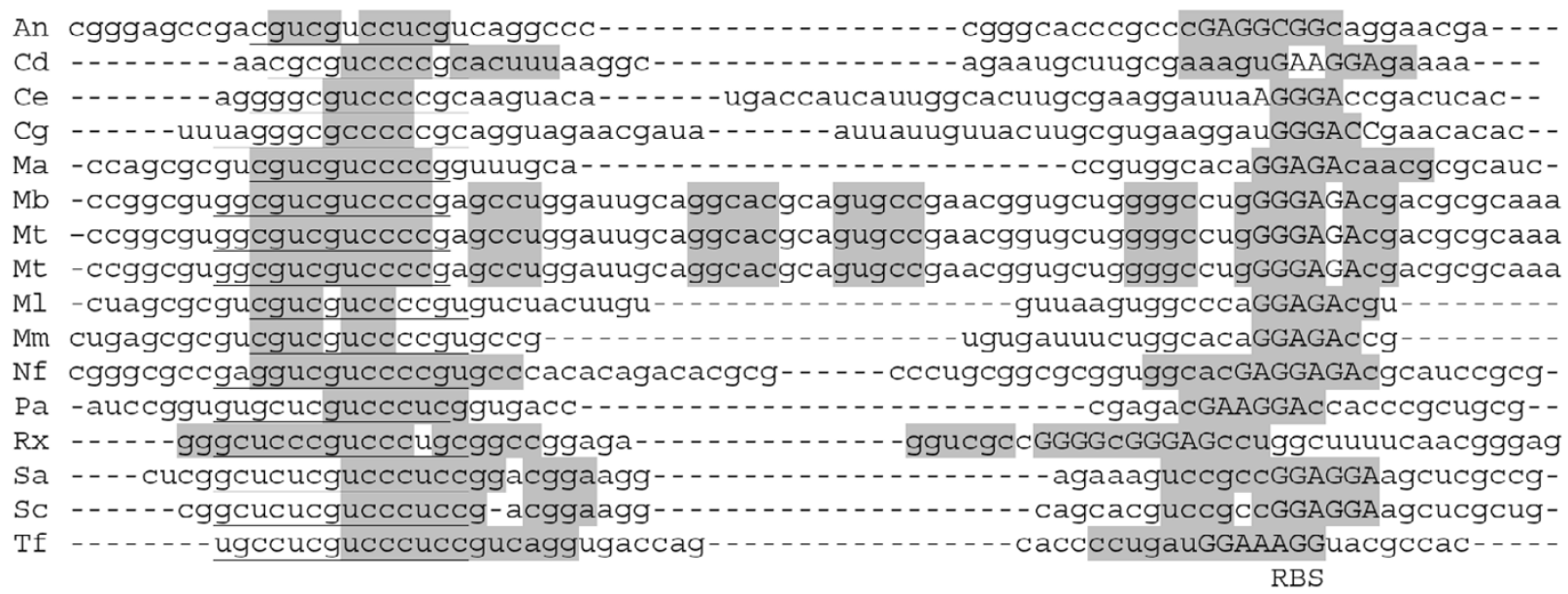

Figure 10

Multiple alignment of T-box structures upstream of the ileS genes. a) Coordinates and protein identifiers of the ileS genes. b) Nucleotide alignment of the 5 ' untranslated regions. T-box hairpins are underlined and T-box sequences are set in capitals. The sequestor hairpin is shaded in grey. Candidate Shine-Dalgarno boxes are set on capitals. Anti-sequestor hairpins are set in bold. 
actinobacterial genome. The main fraction of the coding sequence was subsequently deleted, whereas the structural element was co-opted for regulation of the downstream leuA gene.

\section{Methods}

Genomes of Actinobacteria Actinomyces naeslundii (An), Bifidobacterium longum (Bl), Corynebacterium diphtheriae (Cd), Corynebacterium efficiens (Ce), Corynebacterium glutamicum $(\mathrm{Cg})$, Kineococcus radiotolerans (Kr), Leifsonia xyli (Lx), Mycobacterium avium (Ma), Mycobacterium bovis (Mb), Mycobacterium leprae (Ml), Mycobacterium marinum $(\mathrm{Mm})$, Mycobacterium smegmatis (Ms), Mycobacterium tuberculosis (Rv and Mt), Nocardia farcinica (Nf), Propionibacterium acnes (Pa), Rubrobacter xylanophilus (Rx), Streptomyces avermitilis (Sa), Streptomyces coelicolor (Sc), Thermobifida fusca (Tf), Tropheryma whipplei (Tw) were downloaded from the NCBI web site. We also used sequences of Streptomyces venezuelae (Sv) from [21].

Candidate operons were defined as chains of genes transcribed in the same direction with intergenic regions not exceeding 150 nucleotides. Multiple alignments of genes were used to verify and, if necessary, revise annotated gene starts [33]. The revisions included adding 105 nucleotides (35 codons) to the leuA gene from C. efficiens, adding 27 nucleotides ( 9 codons) of the leuA gene from $T$. fusca, and removing 147 nucleotides (49 codons) of the ileS gene from C. efficiens.

RNA sequence and structure alignments were constructed using MultAlign (A.A. Mironov, personal communication) and the program GL [34]. Search for RNA structural patterns was performed using the PAT program (A.V.Seliverstov, unpublished). Search for conserved sequence fragments was done using the CLIQUE program [35]. Multiple protein sequence alignments were constructed using MultAlign.

\section{Authors' contributions}

AVS and VAL developed algorithms. AVS wrote the programs and performed sequence analysis. HP and AVS identified translational T-boxes. AVS, VAL, and MSG analyzed LEU elements. AVS and MSG performed functional annotation and wrote the paper. VAL and MSG conceived and supervised the project.

\section{Acknowledgements}

We are grateful to Andrei Mironov for the MultAlig program and to K.Yu. Gorbunov for useful discussion. This work was partially supported by grants from ISTC (2766), Howard Hughes Medical Institute (55000309), Russian Academy of Sciences (programs "Molecular and Cellular Biology" and "Origin and Evolution of the Biosphere"), Fund for Support of Russian Science.

\section{References}

I. Henkin TM, Yanofsky C: Regulation by transcription attenuation in bacteria: how RNA provides instructions for transcription termination/antitermination decisions. Bioessays 2002, 24:700-707.

2. Grundy FJ, Henkin TM: The $\mathbf{T}$ box and $\mathbf{S}$ box transcription termination control systems. Front Biosci 2003, 8:d20-3I.

3. Grundy FJ, Henkin TM: Regulation of gene expression by effectors that bind to RNA. Curr Opin Microbiol 2004, 7:|26-131.

4. Mandal M, Breaker RR: Gene regulation by riboswitches. Nat Rev Mol Cell Biol 2004, 5:45I-463.

5. Vitreschak AG, Rodionov DA, Mironov AA, Gelfand MS: Riboswitches: the oldest mechanism for the regulation of gene expression? Trends in Genetics 2004, 20(1):44-50.

6. Yanofsky C: The different roles of tryptophan transfer RNA in regulating trp operon expression in $E$. coli versus $B$. subtilis. Trends in Genetics 2004, 20:367-74.

7. Panina EM, Vitreschak AG, Mironov AA, Gelfand MS: Regulation of aromatic amino acid biosynthesis in gamma-proteobacteria. J Mol Microbiol Biotechnol 200I, 3:529-543.

8. Vitreschak AG, Lyubetskaya EV, Shirshin MA, Gelfand MS, Lyubetsky $V A$ : Attenuation regulation of amino acid biosynthetic operons in proteobacteria: comparative genomics analysis. FEMS Microbiology Letters 2004, 234:357-370.

9. Grundy FJ, Henkin TM: Conservation of a transcription antitermination mechanism in aminoacyl-tRNA synthetase and amino acid biosynthesis genes in gram-positive bacteria. J Mol Biol 1994, 235:798-804.

10. Grundy F], Henkin TM: The $\mathbf{S}$ box regulon: a new global transcription termination control system for methionine and cysteine biosynthesis genes in gram-positive bacteria. Mol Microbiol 1998, 30:737-749.

II. Murphy BA, Grundy FJ, Henkin TM: Prediction of gene function in methylthioadenosine recycling from regulatory signals. J Bacteriol 2002, 184:23|4-23।8.

12. Panina EM, Vitreschak AG, Mironov AA, Gelfand MS: Regulation of biosynthesis and transport of aromatic amino acid in low-GC Gram-positive bacteria. FEMS Microbiol Lett 2003, 222:2I I-220.

13. Sudarsan N, Barrick JE, Breaker RR: Metabolite-binding RNA domains are present in the genes of eukaryotes. RNA 2003, 9:644-7.

14. Rodionov DA, Vitreschak AA, Mironov AA, Gelfand MS: Computational analysis of thiamin regulation in bacteria: Possible mechanisms and new THI-element-regulated genes. J Biol Chem 2003, 277:48949-48959.

15. Henkin TM, Glass BL, Grundy FJ: Analysis of the Bacillus subtilis tyrS gene: conservation of a regulatory sequence in multiple tRNA synthetase genes. J Bacteriol 1992, 174:1299-1306.

16. Barrick JE, Corbino KA, Winkler WC, Nahvi A, Mandal M, Collins J, Lee M, Roth A, Sudarsan N, Jona I, Wickiser JK, Breaker RR: New RNA motifs suggest an expanded scope for riboswitches in bacterial genetic control. Proc Natl Acad Sci USA 2004, I0I:642I-6426.

17. Abreu-Goodger C, Ontiveros-Palacios N, Ciria R, Merino E: Conserved regulatory motifs in bacteria: riboswitches and beyond. Trends Genet 2004, 20:475-9.

18. Vitreschak AA, Rodionov DA, Mironov AA, Gelfand MS: Regulation of riboflavin biosynthesis and transport genes in bacteria by transcriptional and translational attenuation. Nucleic Acids Research 2002, 30:3|4I-315I.

19. Vitreschak AG, Rodionov DA, Mironov AA, Gelfand MS: Regulation of the vitamin $B 12$ metabolism and transport in bacteria by a conserved RNA structural element. RNA 2003, 9: 1084- 1097.

20. Heery DM, Dunican LK: Cloning of the trp gene cluster from a tryptophan-hyperproducing strain of Corynebacterium glutamicum: Identification of a mutation in the trp leader sequence. Applied and Environmental Microbiology 1993, 59:791-799.

21. Lin C, Pradkar AS, Vining LC: Regulation of an antranilate synthase gene in Stryptomyces venezuelae by trp attenuator. Microbiology 1998, I44:197I-1980.

22. Craster HL, Potter CA, Baumberg S: End-product control of branched-chain amino acid biosynthesis genes in Streptomyces coelicolor A3(2): paradoxical relationships between DNA sequence and regulatory phenotype. Microbiology 1999, 145:2375-2384. 
23. Konan KV, Yanofsky C: Rho-dependent transcription termination in the tna operon of Escherichia coli: Roles of the boxA sequence and the rut site. Journal of Bacteriology 2000, 182:398I-3988.

24. Washio T, Sasayama J, Tomita M: Analysis of complete genomes suggests that many prokaryotes do not rely on hairpin formation in transcription termination. Nucleic Acids Research 1998, 26:5456-5463.

25. Unniraman S, Prakash R, Nagaraja V: Conserved economics of transcription termination in eubacteria. Nucleic Acids Research 2002, 30:675-684.

26. Richardson JP: Rho-dependent termination and ATPases in transcript termination. Biochimica et Biophysica Acta 2002, 1577:251-260.

27. Richardson JP: Structural organization of transcription termination factor Rho. The Journal of Biological Chemistry 1996, 27I: $125 I-1254$.

28. Kaplan DL, O'Donne M: Rho factor: transcription termination in four steps dispatch. Current Biology 2003, I3:R7|4-R7|6.

29. Gong $F$, Yanofsky C: Rho's role in transcription attenuation in the tna operon of E. coli. Methods Enzymol 2003, 37 I:383-391.

30. Serganov A, Yuan YR, Pikovskaya O, Polonskaia A, Malinina L, Phan AT, Hobartner C, Micura R, Breaker RR, Patel DJ: Structural basis for discriminative regulation of gene expression by adenineand guanine-sensing mRNAs. Chem Biol 2004, I I:I729-174I.

31. Batey RT, Gilbert SD, Montange RK: Structure of a natural guanine-responsive riboswitch complexed with the metabolite hypoxanthine. Nature 2004, 432:4II-4I5.

32. Koonin EV, llyina TV: Computer-assisted dissection of rolling circle DNA replication. BioSystems 1993, 30:241-268.

33. Baytalulk MV, Gelfand MS, Mironov AA: Exact mapping of prokaryotic gene starts. Briefings in Bioinformatics 2002, 3:18I-194.

34. Gorbunov KYu, Mironov AA, Lyubetsky VA: Search for conserved secondary structures of RNA. Mol Biol 2003, 37:850-860.

35. Lyubetsky VA, Seliverstov AV: Selected algorithms related to finite groups. Information Processes 2003, 3:39-46. (in Russian)

Publish with Bio Med Central and every scientist can read your work free of charge

"BioMed Central will be the most significant development for disseminating the results of biomedical research in our lifetime. "

Sir Paul Nurse, Cancer Research UK

Your research papers will be:

- available free of charge to the entire biomedical community

- peer reviewed and published immediately upon acceptance

- cited in PubMed and archived on PubMed Central

- yours - you keep the copyright

Submit your manuscript here:

http://www.biomedcentral.com/info/publishing_adv.asp
BioMedcentral 